\title{
Collaborer (avec les patients et les familles) afin de faire évoluer la pratique des soins centrés sur le patient
}

\author{
par Lauza Saulnier
}

$\mathrm{L}$ 'hiver approche, le temps est venu de retrouver votre tuque et

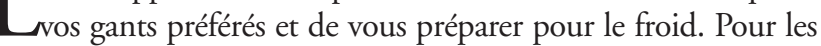
mordus de sports d'hiver, cette saison vivifiante n'arrive pas assez vite. Pour d'autres, l'hiver est synonyme de confort, de bons moments passés avec la famille et les amis de même qu'une période appelant à la réflexion et à la gentillesse. Le changement de saison est le moment par excellence pour adopter un changement de priorité, un nouveau départ ou une transformation.

Le concept de soins centrés sur le patient a reçu une attention croissante au cours des dernières années et il est maintenant considéré comme un aspect essentiel des soins de santé de première qualité. Il nous rappelle les conditions de base nécessaires au soutien apporté aux patients et à leurs proches lorsque nous interagissons avec eux, notamment la gentillesse et une communication efficace. Il nous rappelle aussi l'importance d'encourager le développement de partenariats entre patients, proches et fournisseurs de soins de santé.

Aux États-Unis, l'Institute for Patient-and Family-Centered Care définit les soins centrés sur le patient et la famille comme " une approche pour la planification, la prestation et l'évaluation de soins de santé ancrée au sein d'un partenariat profitable autant aux fournisseurs de soins de santé qu'aux patients et qu'aux familles " [traduction libre]. Les concepts qui la régissent sont le respect et la dignité, le partage de l'information, la participation et la collaboration (www.ipfcc.org/faq.html).

Le programme Qmentum d'Agrément Canada a été révisé afin d'inclure les soins centrés sur l'usager et la famille en tant qu'élément indispensable aux soins de qualité (https://accreditation.ca/fr/soins-centr\%C3\%A9s-sur-1\%E2\%80\%99usager-et-lafamille). Agrément Canada engage les organismes à adopter des pratiques et des principes selon lesquels les fournisseurs n'agissent plus sur l'usager ou pour l'usager, rôle dans lequel la perspective du fournisseur est dominante, mais plutôt avec l'usager. Ainsi, un réel partenariat est établi entre le fournisseur de soins de santé et l'usager. Les nouvelles normes Qmentum citent deux niveaux de mobilisation et de collaboration :

- En partenariat avec l'usager et la famille : où l'équipe collabore directement avec chaque usager et sa famille pour offrir des soins;

- Avec l'apport des usagers et des familles : où une activité est réalisée pour obtenir l'apport des usagers et des familles.
Par souci d'harmonisation avec les organismes clés de santé au Canada, la Société canadienne des pharmaciens d'hôpitaux (SCPH) a intégré une approche axée sur le patient dans sa vision " d'une société dynamique, en constante évolution, qui saura inspirer l'excellence d'une pratique de la pharmacie indispensable aux soins centrés sur le patient dans les hôpitaux et les autres milieux de soins de santé misant sur la collaboration " et dans son plan stratégique de 2015-2018.

Le conseil de la SCPH a approuvé le document « Pratique de la pharmacie dans les hôpitaux et les autres milieux de soins de santé misant sur la collaboration : déclarations de principes » à titre de publication officielle lors de sa réunion d'automne, à Régina, en octobre 2016. Les déclarations dans ce document décrivent un modèle de pratique de la pharmacie souhaité et réalisable qui centre les soins sur le patient. Ce texte servira de pierre d'assise pour le programme Excellence en pharmacie hospitalière, un projet unique et captivant qui orientera le travail des membres vers l'amélioration des résultats cliniques.

D'après un nombre croissant de données, la participation des patients et des familles peut mener à de meilleurs résultats cliniques et à une meilleure performance organisationnelle (http:// www. fcass-cfhi.ca/SearchResultsNews/2014/08/14/evidence-boost-areview-of-research-highlighting-how-patient-engagementcontributes-to-improved-care). Les pharmaciens soutiennent les soins centrés sur le patient et la famille de différentes façons, notamment sur les plans du système et du service ainsi que dans les interactions directes avec les patients et les proches. Afin de s'assurer d'un maximum de bénéfices et de sécurité, il est essentiel que les besoins pharmacothérapeutiques des patients soient pris en charge de manière coordonnée tout au long du continuum des soins.

La SCPH est depuis longtemps reconnue pour faire évoluer la pratique de la pharmacie dans les hôpitaux et les autres milieux de soins de santé misant sur la collaboration. Nous continuerons à canaliser le changement et à soutenir nos membres pour ce qui est de développer et d'entretenir des partenariats efficaces avec les patients et les familles dans le but d'améliorer la qualité et la sécurité des soins et l'expérience qu'en ont les patients.

[Traduction par l'éditeur]

Lauza Saulnier, B. Sc. (Pharm.), ACPR, est présidente et agente de liaison pour la vision de la Société canadienne des pharmaciens d'hôpitaux. 